\title{
Non-Negative Integer Data Type
}

National Cancer Institute

\section{Source}

National Cancer Institute. Non-Negative Integer Data Type. NCI Thesaurus. Code C95670.

A data type comprised of numbers with no fractional part that are constrained to positive numbers as well as zero. 